\title{
Adaptive outer synchronization between two complex delayed dynamical networks via aperiodically intermittent pinning control
}

\author{
Xuqiang Lei ${ }^{a}$, Shuiming Cai ${ }^{\text {a } *}$, Shengqin Jiang ${ }^{b}$, Zengrong Liu ${ }^{\text {c,* }}$ \\ ${ }^{a}$ Faculty of Science, Jiangsu University, Zhenjiang 212013, China \\ ${ }^{\mathrm{b}}$ School of Automation, Southeast University, Nanjing 210096, China \\ ${ }^{c}$ School of Science, Hangzhou Dianzi University, Hangzhou 310018, China
}

\begin{abstract}
In this paper, an aperiodically adaptive intermittent control scheme combined with pinning strategy is proposed for outer synchronization between two general complex delayed dynamical networks. Through constructing a novel piecewise auxiliary function, some sufficient conditions for outer synchronization are derived based on the reduction to absurdity and piecewise analytic method. Different from the periodically intermittent pinning control, here the adaptive intermittent pinning control can be aperiodic. Additionally, an effective pinned-node selection scheme is provided to guide what kinds of nodes should be pinned first. Numerical simulations are finally given to illustrate the effectiveness of the obtained theoretical results.
\end{abstract}

Key words: Complex delayed dynamical network; Outer synchronization; Adaptive intermittent control; Pinning strategy; Aperiodic

\footnotetext{
* Corresponding author :

Email addresses: caishuiming2008@126.com (Shuiming Cai), zrongliu@126.com (Zengrong Liu).
} 


\section{Introduction}

Over the past decade, complex dynamical networks have been extensively investigated in diverse fields of science and engineering, for many natural and manmade systems can be described or modeled by complex dynamical networks [1-4]. In general, a complex dynamical network is a large set of interconnected nodes, where each node is a basic unit with specific dynamics and each edge represents the relationship or connection between them. Typical examples of complex dynamical networks include ecosystems, electricity distribution networks, neural networks, biomolecular networks, and social networks [1-3]. As a typical collective behavior, synchronization of complex dynamical networks has attracted more and more attention. This is partly due to its wide applications in many areas, such as parallel image processing, pattern recognition, secure communication. Up to now, a wide variety of synchronization criteria have been reported for various complex dynamical networks, see [4-12] and relevant references therein.

In reality, there exist two kinds of synchronization phenomena in dynamical networks: inner synchronization and outer synchronization [5,13-17]. Generally speaking, inner synchronization is referred to as synchronization occurring inside a dynamical network [5-9]. This kind of synchronization has been observed in many realistic networks, for instance, synchronized rhythms of cellular clocks within the mammalian suprachiasmatic nucleus [13], and neuronal synchronization in the brain and the entire nervous system [14]. Differing from the inner synchronization, synchronization exhibited between two or more dynamical networks is regarded as outer synchronization [15]. This phenomenon also widely exists in our real world, for example, collective behavior between groups of Drosophila clock neurons [16], balance between predator-prey communities in ecology [15], and the spread of diseases, such as SARS and bird flu, between two communities [17]. Owing to its 
theoretical and practical importance, outer synchronization between two dynamical networks has drawn much attention in recent years [15,18-26]. In [15], Li et al. firstly investigated the outer synchronization between two coupled complex dynamical networks. In [18-20], adaptive control method was adopted to synchronize two complex dynamical networks with or without delay. In [21], with a nonlinear control scheme, the problem of generalized outer synchronization between two different complex dynamical networks was addressed. In [22], Asheghan et al. considered the outer synchronization between two coupled complex networks with fractional-order dynamics. In [23], Wu et al. explored the outer synchronization of uncertain complex delayed dynamical networks with adaptive coupling. In [24], Sun et al. analyzed the effect of noise on the outer synchronization between two unidirectionally coupled complex dynamical networks. In [25], the lag synchronization between two coupled dynamical networks via pinning control was studied. In [26], the outer synchronization between drive-response networks under adaptive impulsive pinning control was discussed.

It should be noted that the control strategies presented in most of previous works on outer synchronization are continuous feedback control methods $[15,18-$ 25] (e.g., state feedback control, adaptive and pinning control), where the control inputs are always assumed to be existed at any time. In reality, this assumption might be rare cases and noneconomic. In comparison with continuous feedback control, intermittent control is a discontinuous control approach. In practice, intermittent control is activated during certain nonzero time intervals, but is off during other time intervals [27]. Thus, intermittent control can reduce the amount of transmitted information and save the control cost. Due to its practical and easy implementation in engineering control, intermittent control has been widely used in engineering fields, including manufacturing, transportation, communication, and 
signal processing $[27,28]$. Recently, a periodically intermittent control scheme has been proposed and applied successfully to study the synchronization problem for complex dynamical networks [28-35,37-41]. In this type of control scheme, the control time is periodic, and in each period, the time is composed of work time and rest time. For instance, the pinning synchronization problem for delayed dynamical networks via periodically intermittent control was considered in [31-33]. In [3436], the cluster synchronization in directed dynamical networks with or without delay via periodically intermittent pinning control was investigated. In [37,38], periodically adaptive intermittent pinning-control strategy was introduced to study the synchronization for directed dynamical networks with node balance and the cluster synchronization for directed heterogeneous dynamical networks, respectively. In [39], based on periodically intermittent adaptive control technique, the outer synchronization between two coupled dynamical networks in finite time was investigated. Zhao et al. [40] explored the problem of outer synchronization between two dynamical networks with delayed dynamical nodes by using periodically adaptive intermittent pinning control. Ma et al. [41] investigated the outer synchronization between two dynamical networks with delayed dynamical nodes and nonlinear delayed coupling via periodically adaptive intermittent pinning control.

However, the requirement of periodicity of intermittent control strategy in previous works [28-41], i.e., each control period is fixed (or equal to each other), may be unreasonable in reality. For instance, the generation of wind power in smart grid is evidently aperiodically intermittent [42-45]. In addition, in practical applications, each control period of intermittent control strategy is needed to be changeable and therefore adjusted in accordance to actual situations. Hence, no matter in the theoretical research and the practical applications, it is imperative to study the synchronization problem for complex dynamical networks via aperiodically (or 
nonperiodically) intermittent control. Very recently, some initial results have been reported on this topic [42-46]. In [42,43], the synchronization problem for complex dynamical networks with linear coupling function as well as nonlinear coupling function via aperiodically intermittent pinning control was addressed, respectively. In [44], the synchronization problem for neural networks with stochastic perturbation was considered under aperiodically intermittent control. In [45], aperiodically intermittent pinning control for the exponential synchronization of linearly coupled networks with delayed dynamical nodes was studied. In [46], the quasisynchronization of nonlinear coupled networks with delayed dynamical nodes and parameter mismatches via aperiodically intermittent pinning control was discussed.

As pointed out in $[40,41,47]$, adaptive scheme can effectively avoid giving larger feedback control gains than those needed in practice. Therefore, it is of great importance to consider the synchronization problem for complex dynamical networks under aperiodically adaptive intermittent pinning control. Unfortunately, there are few results about adaptive approach for aperiodically intermittent pinning control. In $[42,43,45,46]$, to realize synchronization of complex dynamical networks, an aperiodically adaptive intermittent pinning control strategy was proposed. It is worth mentioning that the adaptive rule for intermittent feedback control gain developed in $[42,43,45,46]$ is centralized, which requires the state information of all network nodes (i.e., the global information of the whole network). When a large-scale network is taken into account, this strategy demands huge computing power and communication resources, and hence hard to implement. Hence, a more satisfactory and reasonable adaptive approach is decentralized (or distributed) [37,38], which acquires local information instead of global information of the whole network. To the best of our knowledge, there is still no theoretical results for outer synchronization between two general complex delayed dynamical 
networks via decentralized aperiodically adaptive intermittent pinning control.

Inspired by the above analysis, in this paper, we study the aperiodically intermittent pinning-control problem for outer synchronization between two general complex delayed dynamical networks. By introducing a novel aperiodically adaptive intermittent control scheme combined with pinning strategy and constructing a piecewise auxiliary function, some sufficient conditions for outer synchronization are derived based on the reduction to absurdity and the analytical technique. It is noted that our adaptive scheme is decentralized, and relies on the state information of the controlled node other than all nodes. Moreover, an effective pinned-node selection scheme is given to guide what kinds of nodes should be pinned first. A simple procedure illuminating how to design adaptive nonperiodical intermittent controllers in real applications is also provided. Finally, a numerical example is presented to verify the effectiveness of the theoretical results.

\section{Problem formulation and preliminaries}

Consider a general complex network consisting of $N$ identical time-varying delayed dynamical nodes with linearly diffusive couplings, which is described by:

$$
\dot{x}_{i}(t)=f\left(t, x_{i}(t), x_{i}(t-\tau(t))\right)+c \sum_{j=1}^{N} b_{i j} \Gamma x_{j}(t), \quad i \in \mathfrak{I}=\{1,2, \cdots, N\},
$$

where $x_{i}(t)=\left(x_{i 1}(t), x_{i 2}(t), \ldots, x_{i n}(t)\right)^{\top} \in \mathbb{R}^{n}$ is the state vector of node $i, f: \mathbb{R}^{+} \times$ $\mathbb{R}^{n} \times \mathbb{R}^{n} \rightarrow \mathbb{R}^{n}$ is a continuous vector-valued function, $c>0$ is the coupling strength. $\Gamma=\left(\gamma_{i j}\right) \in \mathbb{R}^{n \times n}$ is the inner connection matrix between two connected nodes, $B=$ $\left(b_{i j}\right) \in \mathbb{R}^{N \times N}$ is the outer coupling matrix reflecting the network topology, in which $b_{i j} \in \mathbb{R}$ is defined as follows: if there exists a directed link from node $j$ to node $i$ ( $j \neq$ $i$ ), then $b_{i j}>0$, otherwise, $b_{i j}=0$. The time-varying delay $\tau(t)$ may be unknown but is bounded by a known constant, i.e., $0 \leq \tau(t) \leq \tau$, in which $\tau(t)$ denotes the internal delay occurring inside the dynamical node [12]. Additionally, the diagonal entries 
of matrix $B$ are defined by $b_{i i}=-\sum_{j=1, j \neq i}^{N} b_{i j}, i \in \mathfrak{I}$, and therefore $\sum_{j=1}^{N} b_{i j}=0, i \in$ $\mathfrak{I}$. The initial values of network (1) are given by $x_{i}(s)=\phi_{i}(s) \in C\left([-\tau, 0], \mathbb{R}^{n}\right), i \in$ $\mathfrak{I}$, where $C\left([-\tau, 0], \mathbb{R}^{n}\right)$ represents the set of all $n$-dimensional continuous functions defined on the interval $[-\tau, 0]$. Note that the outer coupling matrix $B$ is not restricted to be symmetric or irreducible, i.e., the corresponding graph generated by matrix $B$ can be directed, weakly connected and even do not contain any rooted spanning, which is more consistent with a realistic dynamical network.

To make two delayed dynamical networks achieve outer synchronization, we take system (1) as the drive network, and the response network is given by:

$$
\dot{y}_{i}(t)=f\left(t, y_{i}(t), y_{i}(t-\tau(t))\right)+c \sum_{j=1}^{N} b_{i j} \Gamma y_{j}(t)+u_{i}(t), \quad i \in \mathfrak{I},
$$

where $y_{i}(t)=\left(y_{i 1}(t), y_{i 2}(t), \ldots, y_{i n}(t)\right)^{\top} \in \mathbb{R}^{n}$ denotes the state vector of node $i, u_{i}(t)$ is the control input applied to node $i$, and the other notations have the same meanings as those in the drive network. The initial values of system (2) are given by $y_{i}(s)=$ $\varphi_{i}(s) \in C\left([-\tau, 0], \mathbb{R}^{n}\right), i \in \mathfrak{I}$.

Definition 1. The drive network (1) is said to achieve outer synchronization with the response network (2) if, for any initial values $\phi_{i}(s), \varphi_{i}(s) \in C\left([-\tau, 0], \mathbb{R}^{n}\right)$

$$
\lim _{t \rightarrow+\infty}\left\|y_{i}(t)-x_{i}(t)\right\|=0, \quad i \in \mathfrak{I} .
$$

In this paper, the main objective is to apply adaptive intermittent pinningcontrol scheme to make the drive network (1) can achieve outer synchronization with the response network (2). For this purpose, partial nodes of the response network are selected to be controlled. Without loss of generality, let the first $l(1 \leq l<$ $N$ ) nodes of the response network be chosen and pinned, and the control input $u_{i}(t)$ is designed as an adaptive nonperiodical intermittent controller described by

$$
u_{i}(t)=-d_{i}(t)\left(y_{i}(t)-x_{i}(t)\right), \quad 1 \leq i \leq l
$$

in which $d_{i}(t)$ is the adaptive intermittent feedback control gain defined as follows 


$$
d_{i}(t)= \begin{cases}d_{i}(0), & t=0, \\ d_{i}\left(t_{m}+\delta_{m}\right), & t=t_{m+1}, \\ 0, & t_{m}+\delta_{m}<t<t_{m+1},\end{cases}
$$

with the adaptive rule

$$
\dot{d}_{i}(t)=\varepsilon_{i} \exp \left\{\rho_{1} t\right\}\left\|y_{i}(t)-x_{i}(t)\right\|^{2}, \quad t_{m} \leq t \leq t_{m}+\delta_{m},
$$

where $m \in Z^{+}=\{1,2, \cdots\}, \rho_{1}$ is a positive constant, $\varepsilon_{i}>0$ and $d_{i}(0)>0$ for $1 \leq i \leq l$. The time sequence $\left\{t_{m}\right\}_{m=1}^{+\infty}$ satisfies $0=t_{1}<t_{2}<\cdots<t_{m}<\cdots$ and $\lim _{m \rightarrow+\infty} t_{m}=$ $+\infty$. The time span $\left[t_{m}, t_{m+1}\right)$ is the time of the $m$ th period, and $\left(t_{m+1}-t_{m}\right)$ is called the $m$ th control period; $\left[t_{m}, t_{m}+\delta_{m}\right]$ is the $m$ th work time, and $\delta_{m}>0$ is called the $m$ th control width (control duration); $\left(t_{m}+\delta_{m}, t_{m+1}\right)$ is the $m$ th rest time, and $\left(t_{m+1}-t_{m}\right)-\delta_{m}>0$ is called the $m$ th rest width (rest duration). Throughout this paper, without loss of generality, we always assume that all the rest widths $\left(t_{m+1}-t_{m}\right)-\delta_{m}$, $m \in Z^{+}$are bounded.

It can be seen from (5) and (6) that the control time of controller (4) is nonperiodical, and each control period $\left[t_{m}, t_{m+1}\right)$ is composed of "work time $\left[t_{m}, t_{m}+\delta_{m}\right)$ " and "rest time $\left[t_{m}+\delta_{m}, t_{m+1}\right)$ ". The controller is imposed to the network during the work time, but it is removed during the rest time. This type of control scheme is called aperiodically (or nonperiodically) intermittent control [42]. Evidently, the above requirement of $t_{m}$ and $\delta_{m}$ has a large scope. Especially, when $t_{m+1}-t_{m} \equiv \mathrm{T}$ and $\delta_{m} \equiv \delta, m \in Z^{+}$, the intermittent control type becomes the periodic one, which has been widely investigated in the previous works [28-35,37-41].

For convenience, let $\mathrm{T}_{0}=\hat{\mathrm{T}}_{0}=t_{1}, \mathrm{~T}_{m}=t_{m+1}-t_{m}, \hat{\mathrm{T}}_{m}=\sum_{j=0}^{m} \mathrm{~T}_{j}$, and $\theta_{m}=\delta_{m} / \mathrm{T}_{m}$, $m \in Z^{+}$, where $\theta_{m}$ is called the control rate of the $m$ th control period. Then, we have $t_{m}=\hat{\mathrm{T}}_{m-1}$ and $\delta_{m}=\theta_{m} \mathrm{~T}_{m}, m \in Z^{+}$. Defining the synchronization errors as 
$e_{i}(t)=y_{i}(t)-x_{i}(t), i \in \mathfrak{I}$, then we have the following error dynamical system

$$
\left\{\begin{array}{l}
\dot{e}_{i}(t)=\tilde{f}(t,(t-\tau(t)))+c \sum_{j=1}^{N} b_{i j} \Gamma e_{j}(t)-d_{i}(t) e_{i}(t), \quad 1 \leq i \leq l, \quad \hat{\mathrm{T}}_{m-1} \leq t \leq \hat{\mathrm{T}}_{m-1}+\theta_{m} \mathrm{~T}_{m}, \\
\dot{e}_{i}(t)=\tilde{f}(t,(t-\tau(t)))+c \sum_{j=1}^{N} b_{i j} \Gamma e_{j}(t), \quad l+1 \leq i \leq N, \quad \hat{\mathrm{T}}_{m-1} \leq t \leq \hat{\mathrm{T}}_{m-1}+\theta_{m} \mathrm{~T}_{m}, \quad(7) \\
\dot{e}_{i}(t)=\tilde{f}(t,(t-\tau(t)))+c \sum_{j=1}^{N} b_{i j} \Gamma e_{j}(t), \quad i \in \mathfrak{I}, \quad \hat{\mathrm{T}}_{m-1}+\theta_{m} \mathrm{~T}_{m}<t<\hat{\mathrm{T}}_{m},
\end{array}\right.
$$

where $m \in Z^{+}$and $\tilde{f}(t,(t-\tau(t)))=f\left(t, x_{i}(t), x_{i}(t-\tau(t))\right)-f\left(t, y_{i}(t), y_{i}(t-\tau(t))\right)$. Obviously, if the error variables satisfy $\lim _{t \rightarrow+\infty}\left\|e_{i}(t)\right\|=0, i \in \mathfrak{I}$, then the driveresponse networks (1) and (2) can achieve outer synchronization.

As preliminaries, the following statements are necessary.

Assumption 1. For the vector-valued function $f(t, x(t), x(t-\tau(t)))$, there exist two constants $K_{1}$ and $K_{2}$ such that $[29,32]$

$$
\begin{aligned}
& \quad[x(t)-y(t)]^{\top}[f(t, x(t), x(t-\tau(t)))-f(t, y(t), y(t-\tau(t)))] \\
& \leq K_{1}[x(t)-y(t)]^{\top}[x(t)-y(t)] \\
& \quad+K_{2}[x(t-\tau(t))-y(t-\tau(t))]^{\top}[x(t-\tau(t))-y(t-\tau(t))]
\end{aligned}
$$

for any $x(t), y(t) \in \mathbb{R}^{n}$.

Remark 1. Assumption 1 gives some requirements for the dynamics of uncoupled node in network (1). If the function describing node dynamics in network (1) satisfies uniform Lipschitz condition with respect to the time $t$, i.e., $\| f(t, x(t), x(t-$ $\tau(t)))-f(t, y(t), y(t-\tau(t)))\left\|\leq L_{1}\right\| x(t)-y(t)\left\|+L_{2}\right\| x(t-\tau(t))-y(t-\tau(t)) \|$, where $L_{1}, L_{2}>0$ are two positive constants, one can choose $K_{1}=L_{1}+\omega K_{2} / 2$ and $K_{2}=L_{2} /(2 \omega)$ to satisfy Assumption 1 , where $\omega>0$ is a positive constant. In addition, if the function $f(t, x(t), x(t-\tau(t)))$ is reduced to $f(t, x(t))$, then the condition (8) is degenerated to the following condition

$$
[x(t)-y(t)]^{\top}[f(t, x(t))-f(t, y(t))] \leq K_{1}[x(t)-y(t)]^{\top}[x(t)-y(t)] .
$$

It is easy to check that the condition $\left(8^{\prime}\right)$ is equivalent to the so called QUAD 
condition introduced in $[9,34]$. Usually, a vector-valued function $f: \mathbb{R}^{+} \times \mathbb{R}^{n} \rightarrow \mathbb{R}^{n}$ is said to satisfy QUAD condition if, for any $x(t), y(t) \in \mathbb{R}^{n}$,

$$
[x(t)-y(t)]^{\top}[f(t, x(t))-f(t, y(t))-\Delta(x(t)-y(t))] \leq-\varpi[x(t)-y(t)]^{\top}[x(t)-y(t)],
$$

where $\Delta$ is a diagonal matrix of order $n$ and $\varpi$ is a nonnegative scalar. Thus, the condition (8) can be viewed as an generalization of the QUAD condition. Moreover, it has been verified that many well-known chaotic systems with or without delay, such as the Lorenz system, Rössler system, Chen system, Chua's circuit, the delayed Hopfield neural networks and delayed cellular neural networks also satisfy Assumption 1, see $[29,32-34,47,48]$ and the references therein. Similar assumptions have been widely given in the synchronization literature $[29,31-43,45-48]$.

Lemma 1. (Schur complement [38]) The following linear matrix inequality (LMI):

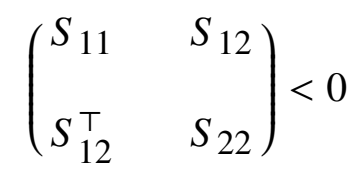

where $S_{11}=S_{11}^{\top}, S_{22}=S_{22}^{\top}$, and $S_{12}$ is a matrix with suitable dimensions, is equivalent to the following condition:

$$
S_{22}<0, \quad S_{11}-S_{12} S_{22}^{-1} S_{12}^{\top}<0
$$

Lemma 2. [38] Assume that $\Xi_{1}$ and $\Xi_{2}$ are two real symmetric matrices in $\mathbb{R}^{N \times N}$. Let $\alpha_{1} \geq \alpha_{2} \geq \cdots \geq \alpha_{N}, \gamma_{1} \geq \gamma_{2} \geq \cdots \geq \gamma_{N}$ and $\lambda_{1} \geq \lambda_{2} \geq \cdots \geq \lambda_{N}$ be eigenvalues of matrices $\Xi_{1}, \Xi_{2}$ and $\Xi_{1}+\Xi_{2}$, respectively. Then, one has $\alpha_{i}+\gamma_{N} \leq \lambda_{i} \leq \alpha_{i}+\gamma_{1}, i \in \mathfrak{I}$.

\section{Main Results}

In this section, by constructing a novel piecewise auxiliary function, some sufficient conditions for outer synchronization between the drive-response networks (1) and (2) under the aperiodically adaptive intermittent pinning control will be derived by employing the Lyapunov stability theory and piecewise analytic technique. 
In the following, let $\varrho_{\min }$ be the minimum eigenvalue of the matrix $\left(\Gamma+\Gamma^{\top}\right) / 2$ and $I_{N}$ be the $N$-dimensional identity matrix. Suppose that $\varrho_{\min } \neq 0$ and $\|\Gamma\|=\varrho_{0}$ $>0$. Denote $\tilde{B}_{s}=\left(\tilde{B}+\tilde{B}^{\top}\right) / 2$, where $\tilde{B}$ is a modified matrix of $B$ via substituting $\left(\varrho_{\min } / \varrho_{0}\right) b_{i i}$ for the diagonal elements $b_{i i}$ of $B$, then $\tilde{B}_{s}$ is a symmetric matrix with nonnegative off-diagonal elements.

Theorem 1. Under Assumption 1, if there exist positive constants $\rho_{1}>2 K_{2}$ and $\rho_{2}>0$ such that the following conditions hold:

(i) $\left(2 K_{1}+\rho_{1}\right) I_{N-l}+2 c \varrho_{0}\left(\tilde{B}_{s}\right)_{l}<0$,

(ii) $2 K_{1} I_{N}+2 c \varrho_{0} \tilde{B}_{s}-\left(\rho_{2}-\rho_{1}\right) I_{N} \leq 0$

(iii) $\lim _{m \rightarrow+\infty}\left[-\lambda\left(\sum_{j=0}^{m-1} \mathrm{~T}_{j}\right)+\rho_{2}\left(\sum_{j=0}^{m-1}\left(1-\theta_{j}\right) \mathrm{T}_{j}\right)\right]=-\infty$,

where $\left(\tilde{B}_{s}\right)_{l}$ is the minor matrix of $\tilde{B}_{s}$ by removing its first $l$ row-column pairs, and $\lambda>0$ is the unique positive root of the equation $\lambda-\rho_{1}+2 K_{2} \exp \{\lambda \tau\}=0$. Then, the drive network (1) can achieve outer synchronization with the response network (2) under the adaptive pinning nonperiodical intermittent controllers (4)-(6).

Proof. Denote $\Phi(z)=z-\rho_{1}+2 K_{2} \exp \{z \tau\}$. Since $\rho_{1}>2 K_{2}>0$, we get $\Phi(0)<0$, $\Phi(+\infty)>0$, and $\Phi^{\prime}(z)>0$. According to the continuity and the monotonicity of $\Phi(z)$, the equation $\Phi(z)=z-\rho_{1}+2 K_{2} \exp \{z \tau\}=0$ has an unique positive root $\lambda>0$.

Introduce a piecewise function defined as

$$
\Psi(t)= \begin{cases}\frac{1}{2} \exp \left\{-\rho_{1} t\right\} \sum_{i=1}^{l} \frac{1}{\varepsilon_{i}}\left(d_{i}(t)-d_{i}^{*}\right)^{2}, & \hat{\mathrm{T}}_{m-1} \leq t \leq \hat{\mathrm{T}}_{m-1}+\theta_{m} \mathrm{~T}_{m}, \\ \frac{1}{2} \exp \left\{-\rho_{1} t\right\} \sum_{i=1}^{l} \frac{1}{\varepsilon_{i}}\left(d_{i}\left(\hat{\mathrm{T}}_{m-1}+\theta_{m} \mathrm{~T}_{m}\right)-d_{i}^{*}\right)^{2}, & \hat{\mathrm{T}}_{m-1}+\theta_{m} \mathrm{~T}_{m}<t<\hat{\mathrm{T}}_{m},\end{cases}
$$

where $m \in Z^{+}, d_{i}^{*}>0$ is a positive constant to be determined later. By (5) and (9), it is easy to check that $\Psi(t)$ is continuous for all $t \geq 0$.

Denote 


$$
P(t)=\frac{1}{2} \sum_{i=1}^{N} e_{i}^{\top}(t) e_{i}(t) \quad \text { and } \quad V(t)=P(t)+\Psi(t) .
$$

Obviously, $P(t)$ is continuous for all $t \geq 0$, and thus $V(t)$ is continuous for all $t \geq 0$.

For $\hat{\mathrm{T}}_{m-1} \leq t \leq \hat{\mathrm{T}}_{m-1}+\theta_{m} \mathrm{~T}_{m}, m \in Z^{+}$, using Assumption 1, the time derivative of $V(t)$ along the trajectories of system (7) can be calculated as follows:

$$
\begin{aligned}
\dot{V}(t)= & \sum_{i=1}^{N} e_{i}^{\top}(t)\left[\tilde{f}(t,(t-\tau(t)))+c \sum_{j=1}^{N} b_{i j} \Gamma e_{j}(t)\right]-\sum_{i=1}^{l} d_{i}(t) e_{i}^{\top}(t) e_{i}(t) \\
& -\frac{\rho_{1}}{2} \exp \left\{-\rho_{1} t\right\} \sum_{i=1}^{l} \frac{1}{\varepsilon_{i}}\left(d_{i}(t)-d_{i}^{*}\right)^{2}+\sum_{i=1}^{l}\left(d_{i}(t)-d_{i}^{*}\right) e_{i}^{\top}(t) e_{i}(t) \\
= & \sum_{i=1}^{N} e_{i}^{\top}(t) \tilde{f}(t,(t-\tau(t)))+c \sum_{i=1}^{N} \sum_{j=1}^{N} b_{i j} e_{i}^{\top}(t) \Gamma e_{j}(t) \\
& -\frac{\rho_{1}}{2} \exp \left\{-\rho_{1} t\right\} \sum_{i=1}^{l} \frac{1}{\varepsilon_{i}}\left(d_{i}(t)-d_{i}^{*}\right)^{2}-\sum_{i=1}^{l} d_{i}^{*} e_{i}^{\top}(t) e_{i}(t) \\
\leq & K_{1} \sum_{i=1}^{N} e_{i}^{\top}(t) e_{i}(t)+K_{2} \sum_{i=1}^{N} e_{i}^{\top}(t-\tau(t)) e_{i}(t-\tau(t))-\sum_{i=1}^{l} d_{i}^{*} e_{i}^{\top}(t) e_{i}(t) \\
& +c \sum_{i=1}^{N} \sum_{j=1, j \neq i}^{N} b_{i j} e_{i}^{\top}(t) \Gamma e_{j}(t)+c \sum_{i=1}^{N} b_{i i} e_{i}^{\top}(t) \Gamma e_{i}(t)-\rho_{1} \Psi(t) \\
\leq & 2 K_{1} P(t)+2 K_{2} P(t-\tau(t))-\rho_{1} \Psi(t)-\sum_{i=1}^{l} d_{i}^{*} e_{i}^{\top}(t) e_{i}(t) \\
& +c \sum_{i=1}^{N} \sum_{j=1, j \neq i}^{N} \varrho_{0} b_{i j}\left\|e_{i}^{\top}(t)\right\|\left\|e_{j}(t)\right\|+c \sum_{i=1}^{N} \varrho_{\min } b_{i i} e_{i}^{\top}(t) e_{i}(t) .
\end{aligned}
$$

Denote $\tilde{e}(t)=\left(\left\|e_{1}(t)\right\|,\left\|e_{2}(t)\right\|, \ldots,\left\|e_{N}(t)\right\|\right)^{\top}$, then one has

$$
\frac{1}{2} \tilde{e}^{\top}(t) \tilde{e}(t)=\frac{1}{2} \sum_{i=1}^{N}\left\|e_{i}(t)\right\|^{2}=\frac{1}{2} \sum_{i=1}^{N} e_{i}^{\top}(t) e_{i}(t)=P(t) .
$$

It follows from (10) that

$$
\begin{aligned}
\dot{V}(t) & \leq K_{1} \tilde{e}^{\top}(t) \tilde{e}(t)+2 K_{2} P(t-\tau(t))+c \varrho_{0} \tilde{e}^{\top}(t) \tilde{B} \tilde{e}(t)-\rho_{1} \Psi(t)-\tilde{e}^{\top}(t) D \tilde{e}(t) \\
& =\tilde{e}^{\top}(t)\left(\left(K_{1}+\rho_{1} / 2\right) I_{N}+c \varrho_{0} \tilde{B}_{s}-D\right) \tilde{e}(t)-\rho_{1} P(t)-\rho_{1} \Psi(t)+2 K_{2} P(t-\tau(t)) \\
& \leq \tilde{e}^{\top}(t)\left(\left(K_{1}+\rho_{1} / 2\right) I_{N}+c \varrho_{0} \tilde{B}_{s}-D\right) \tilde{e}(t)-\rho_{1} V(t)+2 K_{2} V(t-\tau(t))
\end{aligned}
$$

where $D=\operatorname{diag}(d_{1}^{*}, \ldots, d_{l}^{*}, \underbrace{0, \ldots, 0}_{N-l})$. 
Let $\Omega=\left(K_{1}+\rho_{1} / 2\right) I_{N}+c \varrho_{0} \tilde{B}_{s}$ and $\left(K_{1}+\rho_{1} / 2\right) I_{N}+c \varrho_{0} \tilde{B}_{s}-D=\Omega-D=\left(\begin{array}{cc}E-\tilde{D} & S \\ S^{\top} & \Omega_{l}\end{array}\right)$ where $E$ and $S$ are matrices with appropriate dimensions, $\tilde{D}=\operatorname{diag}\left(d_{1}, \ldots, d_{l}\right)$, and $\Omega_{l}$ is the minor matrix of $\Omega$ by removing its first $l(1 \leq l<N)$ row-column pairs, i.e., $\Omega_{l}=c \varrho_{0}\left(\tilde{B}_{s}\right)_{l}+\left(K_{1}+\rho_{1} / 2\right) I_{N-l}$. Evidently, $\Omega_{l}$ is a symmetric matrix, and so, by condition (i), one has $\Omega_{l}<0$. Hence, when $d_{i}^{*}>0,1 \leq i \leq l$ are sufficiently large such that $d_{i}^{*}>\lambda_{\max }\left(E-S \Omega_{l}^{-1} S^{\top}\right), 1 \leq i \leq l$, then it is easy to see that $\Omega-D<0$, which directly follows from Lemma 1 . This combines with (11) to produce

$$
\dot{V}(t) \leq-\rho_{1} V(t)+2 K_{2} V(t-\tau(t)), \quad \hat{\mathrm{T}}_{m-1} \leq t \leq \hat{\mathrm{T}}_{m-1}+\theta_{m} \mathrm{~T}_{m}, m \in Z^{+} .
$$

Similarly, for $\hat{\mathrm{T}}_{m-1}+\theta_{m} \mathrm{~T}_{m}<t<\hat{\mathrm{T}}_{m}, m \in Z^{+}$, with the same approach and using condition (ii), we can obtain

$$
\begin{aligned}
\dot{V}(t)= & e_{i}^{\top}(t)\left[\tilde{f}(t,(t-\tau(t)))+c \sum_{j=1}^{N} b_{i j} \Gamma e_{j}(t)\right] \\
& -\frac{\rho_{1}}{2} \exp \left\{-\rho_{1} t\right\} \sum_{i=1}^{l} \frac{1}{\varepsilon_{i}}\left(d_{i}\left(\hat{\mathrm{T}}_{m-1}+\theta_{m} \mathrm{~T}_{m}\right)-d_{i}^{*}\right)^{2} \\
\leq & 2 K_{1} P(t)+2 K_{2} P(t-\tau(t))+c \sum_{i=1}^{N} \sum_{j=1}^{N} b_{i j} e_{i}^{\top}(t) e_{i}(t)-\rho_{1} \Psi(t) \\
= & \frac{1}{2} \tilde{e}^{\top}(t)\left(2 K_{1} I_{N}+2 c \varrho_{0} \tilde{B}_{s}-\left(\rho_{2}-\rho_{1}\right) I_{N}\right) \tilde{e}(t)+\frac{1}{2}\left(\rho_{2}-\rho_{1}\right) \tilde{e}^{\top}(t) \tilde{e}(t) \\
& +2 K_{2} P(t-\tau(t))-\rho_{1} \Psi(t) \\
\leq & \left(\rho_{2}-\rho_{1}\right) P(t)+2 K_{2} V(t-\tau(t))-\rho_{1} \Psi(t), \\
= & \left(\rho_{2}-\rho_{1}\right) V(t)+2 K_{2} V(t-\tau(t))-\rho_{2} \Psi(t) .
\end{aligned}
$$

Since $\Psi(t) \geq 0$ for all $t \geq 0$ and $\rho_{2}>0$, it follows from (13) that

$$
\dot{V}(t) \leq\left(\rho_{2}-\rho_{1}\right) V(t)+2 K_{2} V(t-\tau(t)), \quad \hat{\mathrm{T}}_{m-1}+\theta_{m} \mathrm{~T}_{m}<t<\hat{\mathrm{T}}_{m}, m \in Z^{+} .
$$

In the following, using (12), (14) and condition (iii), we prove that

$$
\lim _{t \rightarrow+\infty} V(t)=0 .
$$

Take $M_{0}=\sup _{-\tau \leq s \leq 0} V(s), H(t)=\exp \{\lambda t\} V(t)$, where $t \geq-\tau$. Let $W(t)=H(t)-$ $h M_{0}$, where $h>1$ is a constant. Note that $\hat{\mathrm{T}}_{0}=0$, then it is easy to see that 
$W(t)<0, \quad$ for all $t \in\left[-\tau, \hat{\mathrm{T}}_{0}\right]$

Next, we prove that

$$
W(t)<0, \quad \text { for all } t \in\left[\hat{\mathrm{T}}_{0}, \theta_{1} \mathrm{~T}_{1}\right]
$$

We utilize the reduction to absurdity. Otherwise, by (15), there exists a $t^{*} \in\left(\hat{\mathrm{T}}_{0}, \theta_{1} \mathrm{~T}_{1}\right]$ such that

$$
\begin{aligned}
& W\left(t^{*}\right)=0, \quad \dot{W}\left(t^{*}\right) \geq 0 \\
& W(t)<0, \quad-\tau \leq t<t^{*} .
\end{aligned}
$$

Using (12), we have

$$
\begin{aligned}
\dot{W}\left(t^{*}\right) & =\lambda H\left(t^{*}\right)+\exp \left\{\lambda t^{*}\right\} \dot{V}\left(t^{*}\right) \\
& \leq \lambda H\left(t^{*}\right)-\rho_{1} H\left(t^{*}\right)+2 K_{2} \exp \left\{\lambda t^{*}\right\} V\left(t^{*}-\tau\left(t^{*}\right)\right) .
\end{aligned}
$$

From (17) and (18), we can get that

$$
H\left(t^{*}\right)=h M_{0}, \quad H(t)<h M_{0}, \quad-\tau \leq t<t^{*} .
$$

This means that

$$
V\left(t^{*}\right)=h M_{0} \exp \left\{-\lambda t^{*}\right\}, \quad V(t)<h M_{0} \exp \{-\lambda t\}, \quad-\tau \leq t<t^{*}
$$

Hence,

$$
\exp \left\{\lambda t^{*}\right\} V\left(t^{*}-\tau\left(t^{*}\right)\right)<\exp \{\lambda \tau\} h M_{0}=\exp \{\lambda \tau\} H\left(t^{*}\right) .
$$

Combining with (19), we can obtain that

$$
\dot{W}\left(t^{*}\right)<\left(\lambda-\rho_{1}+2 K_{2} \exp \{\lambda \tau\}\right) H\left(t^{*}\right)=0 .
$$

This contradicts the second inequality in (17), which shows that (16) holds. Together with (15), we can get that

$$
V(t)<h M_{0} \exp \{-\lambda t\}, \quad \text { for all } t \in\left[-\tau, \theta_{1} \mathrm{~T}_{1}\right]
$$

Now, we prove that

$$
\Omega(t)=H(t)-h M_{0} \exp \left\{\rho_{2}\left(t-\theta_{1} \mathrm{~T}_{1}\right)\right\}<0, \quad \text { for all } t \in\left(\theta_{1} \mathrm{~T}_{1}, \hat{\mathrm{T}}_{1}\right)
$$

Otherwise, there exists a $t^{* *} \in\left(\theta_{1} \mathrm{~T}_{1}, \hat{\mathrm{T}}_{1}\right)$ such that 


$$
\begin{aligned}
& \Omega\left(t^{* *}\right)=0, \quad \dot{\Omega}\left(t^{* *}\right) \geq 0, \\
& \Omega(t)<0, \quad \theta_{1} \mathrm{~T}_{1}<t<t^{* *} .
\end{aligned}
$$

For $\tau\left(t^{* *}\right)>0$, if $\theta_{1} \mathrm{~T}_{1}<t^{* *}-\tau\left(t^{* *}\right)<t^{* *}$, it follows from (23) and (24) that

$$
\exp \left\{\lambda t^{* *}\right\} V\left(t^{* *}-\tau\left(t^{* *}\right)\right)<\exp \{\lambda \tau\} h M_{0} \exp \left\{\rho_{2}\left(t^{* *}-\theta_{1} \mathrm{~T}_{1}\right)\right\}=\exp \{\lambda \tau\} H\left(t^{* *}\right),
$$

and if $-\tau \leq t^{* *}-\tau\left(t^{* *}\right) \leq \theta_{1} \mathrm{~T}_{1}$, it follows from (21) and (23) that

$$
\exp \left\{\lambda t^{* *}\right\} V\left(t^{* *}-\tau\left(t^{* *}\right)\right)<\exp \{\lambda \tau\} h M_{0} \leq \exp \{\lambda \tau\} H\left(t^{* *}\right)
$$

Therefore, we always have

$$
\exp \left\{\lambda t^{* *}\right\} V\left(t^{* *}-\tau\left(t^{* *}\right)\right)<\exp \{\lambda \tau\} H\left(t^{* *}\right), \quad \text { for } \sigma\left(t^{* *}\right)>0
$$

Hence, according to (14), we get

$$
\begin{aligned}
\dot{\Omega}\left(t^{* *}\right) & =\lambda H\left(t^{* *}\right)+\exp \left\{\lambda t^{* *}\right\} \dot{V}\left(t^{* *}\right)-\rho_{2} h M_{0} \exp \left\{\rho_{2}\left(t^{* *}-\theta_{1} \mathrm{~T}_{1}\right)\right\} \\
& \leq \lambda H\left(t^{* *}\right)+\left(\rho_{2}-\rho_{1}\right) H\left(t^{* *}\right)-\rho_{2} H\left(t^{* *}\right)+2 K_{2} \exp \left\{\lambda t^{* *}\right\} V\left(t^{* *}-\tau\left(t^{* *}\right)\right) \\
& <\left(\lambda-\rho_{1}+2 K_{2} \exp \{\lambda \tau\}\right) H\left(t^{*}\right)=0,
\end{aligned}
$$

which contradicts the second inequality in (23). Therefore (22) holds, i.e.,

$$
H(t)<h M_{0} \exp \left\{\rho_{2}\left(t-\theta_{1} \mathrm{~T}_{1}\right)\right\} \leq h M_{0} \exp \left\{\rho_{2}\left(1-\theta_{1}\right) \mathrm{T}_{1}\right\}, \quad \text { for all } t \in\left(\theta_{1} \mathrm{~T}_{1}, \hat{\mathrm{T}}_{1}\right) \text {. }
$$

Combining with inequalities (15) and (16), we get

$$
H(t)<h M_{0} \exp \left\{\rho_{2}\left(1-\theta_{1}\right) \mathrm{T}_{1}\right\}, \quad \text { for all } t \in\left[-\tau, \hat{\mathrm{T}}_{1}\right) \text {. }
$$

Similar to the proofs of (16) and (22), we can prove that

$$
H(t)<h M_{0} \exp \left\{\rho_{2}\left(1-\theta_{1}\right) \mathrm{T}_{1}\right\}, \quad \text { for all } t \in\left[\hat{\mathrm{T}}_{1}, \hat{\mathrm{T}}_{1}+\theta_{2} \mathrm{~T}_{2}\right]
$$

and

$$
\begin{aligned}
H(t) & <h M_{0} \exp \left\{\rho_{2}\left(1-\theta_{1}\right) \mathrm{T}_{1}\right\} \exp \left\{\rho_{2}\left(t-\hat{\mathrm{T}}_{1}-\theta_{2} \mathrm{~T}_{2}\right)\right\} \\
& =h M_{0} \exp \left\{\rho_{2}\left(t-\left(\theta_{1} \mathrm{~T}_{1}+\theta_{2} \mathrm{~T}_{2}\right)\right)\right\}, \quad \text { for all } t \in\left(\hat{\mathrm{T}}_{1}+\theta_{2} \mathrm{~T}_{2}, \hat{\mathrm{T}}_{2}\right) .
\end{aligned}
$$

Furthermore, we can derive that for all $t \in\left[\hat{\mathrm{T}}_{2}, \hat{\mathrm{T}}_{2}+\theta_{3} \mathrm{~T}_{3}\right]$

$$
H(t)<h M_{0} \exp \left\{\rho_{2}\left(\left(1-\theta_{1}\right) \mathrm{T}_{1}+\left(1-\theta_{2}\right) \mathrm{T}_{2}\right)\right\},
$$

and for all $t \in\left(\hat{\mathrm{T}}_{2}+\theta_{3} \mathrm{~T}_{3}, \hat{\mathrm{T}}_{3}\right)$ 


$$
H(t)<h M_{0} \exp \left\{\rho_{2}\left(t-\left(\theta_{1} \mathrm{~T}_{1}+\theta_{2} \mathrm{~T}_{2}+\theta_{3} \mathrm{~T}_{3}\right)\right)\right\}
$$

By mathematical induction, we can derive the following estimation of $H(t)$ for any positive integer $m$.

For all $\hat{\mathrm{T}}_{m-1} \leq t \leq \hat{\mathrm{T}}_{m-1}+\theta_{m} \mathrm{~T}_{m}, m \in Z^{+}$,

$$
H(t)<h M_{0} \exp \left\{\rho_{2}\left(\sum_{j=0}^{m-1}\left(1-\theta_{j}\right) \mathrm{T}_{j}\right)\right\} .
$$

And for all $\hat{\mathrm{T}}_{m-1}+\theta_{m} \mathrm{~T}_{m}<t<\hat{\mathrm{T}}_{m}, m \in Z^{+}$,

$$
H(t)<h M_{0} \exp \left\{\rho_{2}\left(t-\left(\sum_{j=0}^{m} \theta_{j} \mathrm{~T}_{j}\right)\right)\right\} \leq h M_{0} \exp \left\{\rho_{2}\left(\sum_{j=0}^{m}\left(1-\theta_{j}\right) \mathrm{T}_{j}\right)\right\}
$$

Recalling that $H(t)=\exp \{\lambda t\} V(t)$, it follows from (25) and (26) that for all $\hat{\mathrm{T}}_{m-1} \leq t \leq \hat{\mathrm{T}}_{m-1}+\theta_{m} \mathrm{~T}_{m}, m \in Z^{+}$,

$$
\begin{aligned}
V(t) & <h M_{0} \exp \{-\lambda t\} \exp \left\{\rho_{2}\left(\sum_{j=0}^{m-1}\left(1-\theta_{j}\right) \mathrm{T}_{j}\right)\right\} \\
& \leq h M_{0} \exp \left\{-\lambda\left(\sum_{j=0}^{m-1} \mathrm{~T}_{j}\right)+\rho_{2}\left(\sum_{j=0}^{m-1}\left(1-\theta_{j}\right) \mathrm{T}_{j}\right)\right\},
\end{aligned}
$$

and for all $\hat{\mathrm{T}}_{m-1}+\theta_{m} \mathrm{~T}_{m}<t<\hat{\mathrm{T}}_{m}, m \in Z^{+}$,

$$
\begin{aligned}
V(t) & <h M_{0} \exp \{-\lambda t\} \exp \left\{\rho_{2}\left(\sum_{j=0}^{m}\left(1-\theta_{j}\right) \mathrm{T}_{j}\right)\right\} \\
& <h M_{0} \exp \left\{\rho_{2}\left(1-\theta_{m}\right) \mathrm{T}_{m}\right\} \exp \left\{-\lambda\left(\sum_{j=0}^{m-1} \mathrm{~T}_{j}\right)+\rho_{2}\left(\sum_{j=0}^{m-1}\left(1-\theta_{j}\right) \mathrm{T}_{j}\right)\right\} .
\end{aligned}
$$

Since all the rest widths $\left(\mathrm{T}_{m}-\delta_{m}\right), m \in Z^{+}$, are bounded, we can assume that $\sup _{m \in Z^{+}}\left\{\mathrm{T}_{m}-\delta_{m}\right\}=\varpi_{0}$, where $\varpi_{0}>0$ is a positive constant. Denote $\Upsilon_{0}=$ $h M_{0} \exp \left\{\rho_{2} \varpi_{0}\right\}$, we have from (27) and (28) that

$$
V(t)<\Upsilon_{0} \exp \left\{-\lambda\left(\sum_{j=0}^{m-1} \mathrm{~T}_{j}\right)+\rho_{2}\left(\sum_{j=0}^{m-1}\left(1-\theta_{j}\right) \mathrm{T}_{j}\right)\right\}, \quad \text { for all } t \geq 0 .
$$

According to condition (iii), one has

$$
\lim _{t \rightarrow+\infty} V(t)=0 .
$$


which means that $\lim _{t \rightarrow+\infty}\left\|e_{i}(t)\right\|=0$, for all $i \in \mathfrak{I}$. The proof is thus completed.

Remark 2. As $\delta_{m} \rightarrow \mathrm{T}_{m}$ or $\theta_{m} \rightarrow 1, m \in Z^{+}$, the aperiodically adaptive intermittent pinning control is degenerated to the general continuous-time adaptive pinning control, which has been studied in previous works such as $[47,48]$. For this case, conditions (i) and (ii) ensure outer synchronization between the drive-response networks (1) and (2) since condition (iii) is automatically satisfied.

Remark 3. It is clear from (5) and (6) that the adaptive intermittent feedback control gains $d_{i}(t)$ for $1 \leq i \leq l$ are monotonically increasing during the work time but identically equal to zeros during the rest time. When the outer synchronization is achieved, they converge to some positive constants during each work time. In the following section, numerical simulations will be given to illustrate this point.

Let $\eta_{0}=2 K_{1}+2 c \varrho_{0} \lambda_{\max }\left(\tilde{B}_{s}\right)$ and select $\rho_{2}=\rho_{1}+\eta_{0}>0$, then condition (ii) holds. In addition, by Lemma 2 , we get that $\lambda_{\max }\left(\left(2 K_{1}+\rho_{1}\right) I_{N-l}+2 c \varrho_{0}\left(\tilde{B}_{s}\right)_{l}\right) \leq 2 K_{1}+\rho_{1}+$ $2 c \varrho_{0} \lambda_{\max }\left(\left(\tilde{B}_{s}\right)_{l}\right)$. Hence, the following corollary is derived directly from Theorem 1 .

Corollary 1. Under Assumption 1 , if there exists a positive constant $\rho_{1}>2 K_{2}$ such that the following conditions hold:

(i') $\lambda_{\max }\left(\left(\tilde{B}_{s}\right)_{l}\right)<\frac{2 K_{1}+\rho_{1}}{2 c \varrho_{0}}$,

(ii') $\lim _{m \rightarrow+\infty}\left[-\lambda\left(\sum_{j=0}^{m-1} \mathrm{~T}_{j}\right)+\left(\rho_{1}+\eta_{0}\right)\left(\sum_{j=0}^{m-1}\left(1-\theta_{j}\right) \mathrm{T}_{j}\right)\right]=-\infty$,

where $\left(\tilde{B}_{s}\right)_{l}$ is the minor matrix of $\tilde{B}_{s}$ by removing its first $l$ row-column pairs, and $\lambda>0$ is the unique positive root of the equation $\lambda-\rho_{1}+2 K_{2} \exp \{\lambda \tau\}=0$. Then, the drive network (1) can achieve outer synchronization with the response network (2) under the adaptive pinning nonperiodical intermittent controllers (4)-(6).

Suppose that $\rho_{1}$ is given as $\rho_{1}^{*}>2 K_{2}$. Substituting $\rho_{1}=\rho_{1}^{*}$ into the equation $\lambda-\rho_{1}+2 K_{2} \exp \{\lambda \tau\}=0$ yields $\lambda=\psi\left(\rho_{1}^{*}\right)$, then we can obtain the following result. 
Corollary 2. Suppose that Assumption 1 is satisfied and $\rho_{1}$ is given as $\rho_{1}^{*}>2 K_{2}$. Then, the drive network (1) can achieve outer synchronization with the response network (2) under the adaptive pinning nonperiodical intermittent controllers (4)(6) if the following conditions hold:

$$
\begin{aligned}
& \text { (i') } \lambda_{\max }\left(\left(\tilde{B}_{s}\right)_{l}\right)<\frac{2 K_{1}+\rho_{1}^{*}}{2 c \varrho_{0}}, \\
& \text { (ii') } \lim _{m \rightarrow+\infty}\left[-\psi\left(\rho_{1}^{*}\right)\left(\sum_{j=0}^{m-1} \mathrm{~T}_{j}\right)+\left(\rho_{1}^{*}+\eta_{0}\right)\left(\sum_{j=0}^{m-1}\left(1-\theta_{j}\right) \mathrm{T}_{j}\right)\right]=-\infty,
\end{aligned}
$$

where $\left(\tilde{B}_{s}\right)_{l}$ is the minor matrix of $\tilde{B}_{s}$ by removing its first $l$ row-column pairs.

Remark 4. Corollary 2 gives a low-dimensional pinning criterion guaranteeing the drive-response networks (1) and (2) can achieve outer synchronization under the aperiodically adaptive intermittent pinning control. Unfortunately, this criterion does not show any hint of how to find $l$ pinned nodes such that pinning condition (i') in Corollary 2 holds and how to locate them in the response network. As is well known, for a general dynamical network, how to choose appropriate pinned nodes is still a challenging problem $[47,48]$. Obviously, to make pinning condition (i') in Corollary 2 hold, at least we need to choose $l$ pinned candidates such that $\lambda_{\max }\left(\left(\tilde{B}_{s}\right)_{l}\right)<0$. Denote $\mathcal{G}$ as the underlying digraph of matrix $B$. In [47], it was shown that if the nodes whose out-degrees are bigger than their in-degrees in the digraph $\mathcal{G}$ are selected as pinned candidates, then one may have $\lambda_{\max }\left(\left(\tilde{B}_{s}\right)_{l}\right) \leq 0$. Inspired by this idea, we can perform the following steps to select pinned nodes for the response network (2) such that pinning condition (i') in Corollary 2 holds:

(1) For the underlying digraph $\mathcal{G}$ of the outer coupling matrix $B$, denote $\operatorname{Deg}_{\text {in }}(i)$ and $\operatorname{Deg}_{\text {out }}(i)$ as the in-degree and out-degree of node $i$ in $\mathcal{G}$, respectively, i.e.,

$$
\operatorname{Deg}_{\text {in }}(i)=\sum_{j=1, j \neq i}^{N} b_{i j} \quad \text { and } \operatorname{Deg}_{\text {out }}(i)=\sum_{j=1, j \neq i}^{N} b_{j i} \text {. }
$$

(2) Let $\operatorname{Deg}_{\text {diff }}(i)=\operatorname{Deg}_{\text {out }}(i)-\operatorname{Deg}_{\text {in }}(i)$ be the degree-difference between the out-degree and in-degree of node $i$ in $\mathcal{G}, i \in \mathfrak{I}$. 
(3) Select the nodes with zero in-degrees as pinned nodes first because their states are not influenced by others.

(4) Rearrange the remaining nodes of the response network in descending order according to their degree-differences (for those nodes with the same degreedifferences, in descending order according to their out-degrees). According to the degree-differences sequence, increase the number of nodes to be pinned until condition ( $\mathrm{i}^{\prime}$ ) of Corollary 2 is satisfied. Additionally, it can be deduced that the number of nodes to be selected and pinned is at least $l_{0}$, where $l_{0}$ satisfies

$$
\lambda_{\max }\left(\left(\tilde{B}_{s}\right)_{l_{0}-1}\right) \geq \frac{2 K_{1}+\rho_{1}^{*}}{2 c \varrho_{0}} \quad \text { and } \quad \lambda_{\max }\left(\left(\tilde{B}_{s}\right)_{l_{0}}\right)<\frac{2 K_{1}+\rho_{1}^{*}}{2 c \varrho_{0}} .
$$

In the following, for simplifying the validation of condition (ii') of Corollary 2, some special cases will be discussed.

Case 1: Periodically adaptive intermittent pinning-control scheme.

When each control period and each control rate are fixed, i.e., $\mathrm{T}_{m} \equiv \mathrm{T}$ and $\theta_{m} \equiv \theta$ for all $m \in Z^{+}$, where $\mathrm{T}$ and $\theta$ are both positive constants, then the control type becomes periodically adaptive intermittent pinning control, which has been investigated in $[40,41]$. For this case, it is easy to observe that $-\psi\left(\rho_{1}^{*}\right)\left(\sum_{j=0}^{m-1} \mathrm{~T}_{j}\right)+\left(\rho_{1}^{*}+\eta_{0}\right)\left(\sum_{j=0}^{m-1}\left(1-\theta_{j}\right) \mathrm{T}_{j}\right)=(m-1)\left(-\psi\left(\rho_{1}^{*}\right)+\left(\rho_{1}^{*}+\eta_{0}\right)(1-\theta)\right) \mathrm{T}$. Hence, if $-\psi\left(\rho_{1}^{*}\right)+\left(\rho_{1}^{*}+\eta_{0}\right)(1-\theta)<0$, then condition (ii') in Corollary holds. Consequently, we can derive the following result from Corollary 2.

Corollary 3. Suppose that Assumption 1 is satisfied and $\rho_{1}$ is given as $\rho_{1}^{*}>2 K_{2}$. Then, the drive network (1) can achieve outer synchronization with the response network (2) under the periodically adaptive intermittent pinning control if the following conditions hold:

(i') $\lambda_{\max }\left(\left(\tilde{B}_{s}\right)_{l}\right)<\frac{2 K_{1}+\rho_{1}^{*}}{2 c \varrho_{0}}$, 
(ii') $1-\frac{\psi\left(\rho_{1}^{*}\right)}{\rho_{1}^{*}+\eta_{0}}<\theta<1$,

where $\left(\tilde{B}_{s}\right)_{l}$ is the minor matrix of $\tilde{B}_{s}$ by removing its first $l$ row-column pairs.

Case 2: Aperiodically adaptive intermittent pinning-control scheme with fixed control rate.

When each control rate is fixed, i.e., $\theta_{m} \equiv \theta_{\mathrm{eq}}$ for all $m \in Z^{+}$, where $\theta_{\mathrm{eq}}$ is a positive constant, then the control type is aperiodically adaptive intermittent pinning control with fixed control rate but non-fixed control period (called aperiodically adaptive intermittent pinning control with fixed control rate). Obviously, Case 1 can be viewed as a special case for this type of adaptive intermittent control. For this case, one can see that

$-\psi\left(\rho_{1}^{*}\right)\left(\sum_{j=0}^{m-1} \mathrm{~T}_{j}\right)+\left(\rho_{1}^{*}+\eta_{0}\right)\left(\sum_{j=0}^{m-1}\left(1-\theta_{j}\right) \mathrm{T}_{j}\right)=\left(-\psi\left(\rho_{1}^{*}\right)+\left(\rho_{1}^{*}+\eta_{0}\right)\left(1-\theta_{\mathrm{eq}}\right)\right)\left(\sum_{j=0}^{m-1} \mathrm{~T}_{j}\right)$.

Therefore, if $-\psi\left(\rho_{1}^{*}\right)+\left(\rho_{1}^{*}+\eta_{0}\right)\left(1-\theta_{\mathrm{eq}}\right)<0$, then condition (ii') in Corollary 2 holds. Consequently, we have the following result from Corollary 2.

Corollary 4. Suppose that Assumption 1 is satisfied and $\rho_{1}$ is given as $\rho_{1}^{*}>2 K_{2}$. Then, the drive network (1) can achieve outer synchronization with the response network (2) under the aperiodically adaptive intermittent pinning control with fixed control rate if the following conditions hold:

(i') $\lambda_{\max }\left(\left(\tilde{B}_{s}\right)_{l}\right)<\frac{2 K_{1}+\rho_{1}^{*}}{2 c \varrho_{0}}$,

(ii') $1-\frac{\psi\left(\rho_{1}^{*}\right)}{\rho_{1}^{*}+\eta_{0}}<\theta_{\mathrm{eq}}<1$,

where $\left(\tilde{B}_{s}\right)_{l}$ is the minor matrix of $\tilde{B}_{s}$ by removing its first $l$ row-column pairs.

Case 3: Aperiodically adaptive intermittent pinning-control scheme.

Suppose that $\inf _{m \in Z^{+}}\left\{\theta_{m}\right\}=\theta_{\text {inf }}>0$, where $\theta_{\text {inf }}$ is a positive constant. Then

$-\psi\left(\rho_{1}^{*}\right)\left(\sum_{j=0}^{m-1} \mathrm{~T}_{j}\right)+\left(\rho_{1}^{*}+\eta_{0}\right)\left(\sum_{j=0}^{m-1}\left(1-\theta_{j}\right) \mathrm{T}_{j}\right) \leq\left(-\psi\left(\rho_{1}^{*}\right)+\left(\rho_{1}^{*}+\eta_{0}\right)\left(1-\theta_{\text {inf }}\right)\right)\left(\sum_{j=0}^{m-1} \mathrm{~T}_{j}\right)$. 
Thus, if $-\psi\left(\rho_{1}^{*}\right)+\left(\rho_{1}^{*}+\eta_{0}\right)\left(1-\theta_{\text {inf }}\right)<0$, then condition (ii' $)$ in Corollary 2 holds. Evidently, this adaptive intermittent control type takes the aforementioned two types of adaptive intermittent control (Case 1 and Case 2) as special cases. For this case, we can obtain the following corollary from Corollary 2 .

Corollary 5. Suppose that Assumption 1 is satisfied and $\rho_{1}$ is given as $\rho_{1}^{*}>2 K_{2}$. Then, the drive network (1) can achieve outer synchronization with the response network (2) under the aperiodically adaptive intermittent pinning control if the following conditions hold:

$$
\begin{aligned}
& \text { (i') } \lambda_{\max }\left(\left(\tilde{B}_{s}\right)_{l}\right)<\frac{2 K_{1}+\rho_{1}^{*}}{2 c \varrho_{0}}, \\
& \text { (ii') } 1-\frac{\psi\left(\rho_{1}^{*}\right)}{\rho_{1}^{*}+\eta_{0}}<\theta_{\mathrm{inf}}<1,
\end{aligned}
$$

where $\left(\tilde{B}_{s}\right)_{l}$ is the minor matrix of $\tilde{B}_{s}$ by removing its first $l$ row-column pairs.

Remark 5. From Corollary 5, one can see that only the control rate $\theta_{\text {inf }}$, rather than either the control width $\delta_{m}$ or the control period $\mathrm{T}_{m}$, affects the control performance. It indicates that, for achieving the outer synchronization, each control period $\mathrm{T}_{m}$, $m \in Z^{+}$can be arbitrarily selected according to the actual requirement, only if condition (ii') in Corollary 5 holds. This facilitates the potential practical applications of the theoretical results in engineering fields.

Remark 6. In [28-41], the synchronization problem for complex dynamical networks via intermittent control or adaptive intermittent control was discussed. However, the designed controllers in [28-41] are periodically intermittent with fixed control period and control width (i.e., $\mathrm{T}_{m} \equiv \mathrm{T}$ and $\delta_{m} \equiv \delta$ for all $m \in Z^{+}$). Obviously, this requirement may be unreasonable in reality. In this paper, the outer synchronization between two general delayed dynamical networks (1) and (2) is studied via aperiodically adaptive intermittent pinning control with non-fixed both control period and control width. Hence, our theoretical results expand the scope 
of practical applications of intermittent control strategy proposed in [28-41].

Remark 7. In [40], Zhao et al. studied the outer synchronization between two complex networks with delayed dynamical nodes via adaptive intermittent pinning control. However, the adaptive intermittent pinning control adopted in [40] is periodic; in addition, the time delay considered in [40] is constant delay. In this paper, by developing an aperiodically adaptive intermittent control technique combined with pinning strategy, outer synchronization between two complex networks with delayed dynamical nodes is further investigated, where the time delay can be constant or time-varying, and even non-differentiable. Hence, the results derived here generalize the results in [40].

Remark 8. In $[42,43,45,46]$, an adaptive scheme for aperiodically intermittent pinning control was proposed, where the adaptive rule is centralized, and requires the state information of all network nodes. Evidently, it is hard and costly to implement for a network of large scale. In this paper, a new aperiodically adaptive intermittent pinning-control strategy is introduced. It can be seen from (4)-(6) that the designed controller is dependent on the state information of the controlled node other than all nodes. That is to say, the adaptive strategy for aperiodically intermittent pinning control developed in this paper is decentralized. Our theoretical results is hence more practically applicable than those in $[42,43,45,46]$.

Remark 9. To show how to design suitable adaptive nonperiodical intermittent controllers in real-world applications for realizing the outer synchronization, we take example for the application of Corollary 5, the following procedures are preformed:

Step 1. For a given $\rho_{1}^{*}>2 K_{2}$, according to Remark 4, determine $l$ pinned-nodes such that condition (i') of Corollary 5 is satisfied.

Step 2. Calculate the value of $\psi\left(\rho_{1}^{*}\right)$, and then select control rates $\theta_{m}, m \in Z^{+}$ such that condition (ii') of Corollary 5 holds. 
Step 3. Choose control periods $\mathrm{T}_{m}, m \in Z^{+}$according to the actual requirement.

Step 4. Based on the above chosen pinned-nodes, $\theta_{m}, \mathrm{~T}_{m}$, design the adaptive pinning nonperiodical intermittent controllers described in (4)-(6).

\section{Numerical simulations}

In this section, we provide a numerical example to illustrate the effectiveness of the above theoretical results. The delayed Chua oscillator is taken as the node dynamics of the drive-response networks (1) and (2), which is described by $[31,33]$

$$
\dot{x}(t)=f(t, x(t), x(t-\tau(t)))=A x(t)+g_{1}(x(t))+g_{2}(x(t-\tau(t))),
$$

where $x(t)=\left(x_{1}(t), x_{2}(t), x_{3}(t)\right)^{\top} \in \mathbb{R}^{3}, g_{1}(x(t))=\left(-\frac{1}{2} \alpha_{0}\left(a_{1}-a_{2}\right)\left(\left|x_{1}(t)+1\right|-\mid x_{1}(t)-\right.\right.$ 1| $), 0,0)^{\top} \in \mathbb{R}^{3}, g_{2}(x(t-\tau(t)))=\left(0,0,-\beta_{0} \pi_{0} \sin \left(v_{0} x_{1}(t-\tau(t))\right)\right)^{\top} \in \mathbb{R}^{3}, A=$ $\left(\begin{array}{ccc}-\alpha_{0}\left(1+a_{2}\right) & \alpha_{0} & 0 \\ 1 & -1 & 1 \\ 0 & -\beta_{0} & -\omega_{0}\end{array}\right)$, and $\alpha_{0}=10, \beta_{0}=17.53, \omega_{0}=0.1636, a_{1}=-1.4325, a_{2}=-0.7831$, $v_{0}=0.5, \pi_{0}=0.2$, and $\tau(t)=0.02$. As indicated in Fig. 1, the delayed Chua oscillator (30) has a chaotic attractor. In addition, it is easy to verify that

$$
\begin{aligned}
& {[x(t)-y(t)]^{\top}[f(t, x(t), x(t-\tau(t)))-f(t, y(t), y(t-\tau(t)))] } \\
\leq & \frac{1}{2}[x(t)-y(t)]^{\top}\left(A+A^{\top}\right)[x(t)-y(t)]+\left|\alpha_{0}\left(a_{1}-a_{2}\right)\right|\left[x_{1}(t)-y_{1}(t)\right]^{2} \\
& +\beta_{0} \pi_{0} v_{0}\left|x_{3}(t)-y_{3}(t)\right|\left|x_{1}(t-\tau(t))-y_{1}(t-\tau(t))\right| \\
\leq & \lambda_{\max }(\hat{A})[x(t)-y(t)]^{\top}[x(t)-y(t)] \\
& +\left(\beta_{0} \pi_{0} v_{0}\right) /\left(2 \kappa_{1}\right)[x(t-\tau(t))-y(t-\tau(t))]^{\top}[x(t-\tau(t))-y(t-\tau(t))] \\
= & K_{1}[x(t)-y(t)]^{\top}[x(t)-y(t)] \\
& +K_{2}[x(t-\tau(t))-y(t-\tau(t))]^{\top}[x(t-\tau(t))-y(t-\tau(t))],
\end{aligned}
$$

where $\hat{A}=\left(A+A^{\top}\right) / 2+\operatorname{diag}\left(\left|\alpha_{0}\left(a_{1}-a_{2}\right)\right|, 0, \kappa_{1}\left(\beta_{0} \pi_{0} v_{0}\right) / 2\right)$, and $K_{1}=\lambda_{\max }(\tilde{A}), K_{2}=$ $\left(\beta_{0} \pi_{0} v_{0}\right) /\left(2 \kappa_{1}\right)$ can be determined by choosing an appropriate parameter $\kappa_{1}>0$. Therefore, Assumption 1 is satisfied.

For simplicity, we set $\Gamma=\operatorname{diag}(1,1.1,1)$ and $c=15$. Here the network structure of the drive-response networks (1) and (2) is assumed to obey the scale-free 


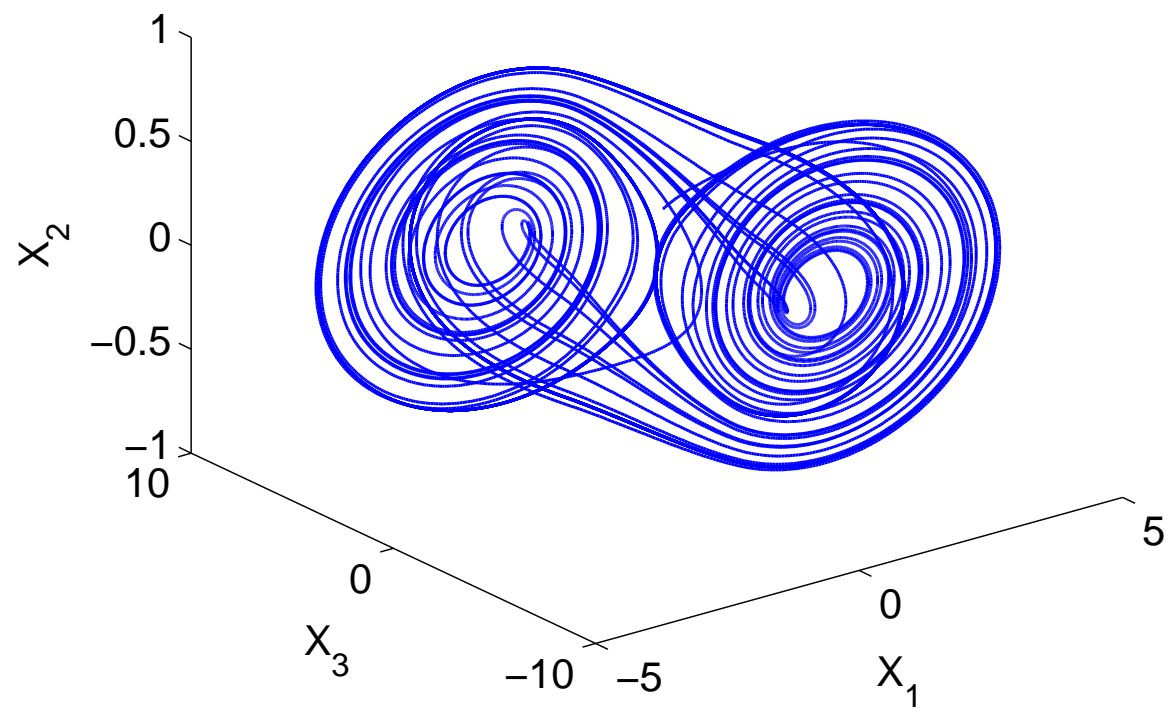

Fig. 1. Chaotic attractor generated by system (30) with initial values $x_{1}(s)=0.5$, $x_{2}(s)=0.2$ and $x_{3}(s)=1.0$ for $s \in[-0.02,0]$.

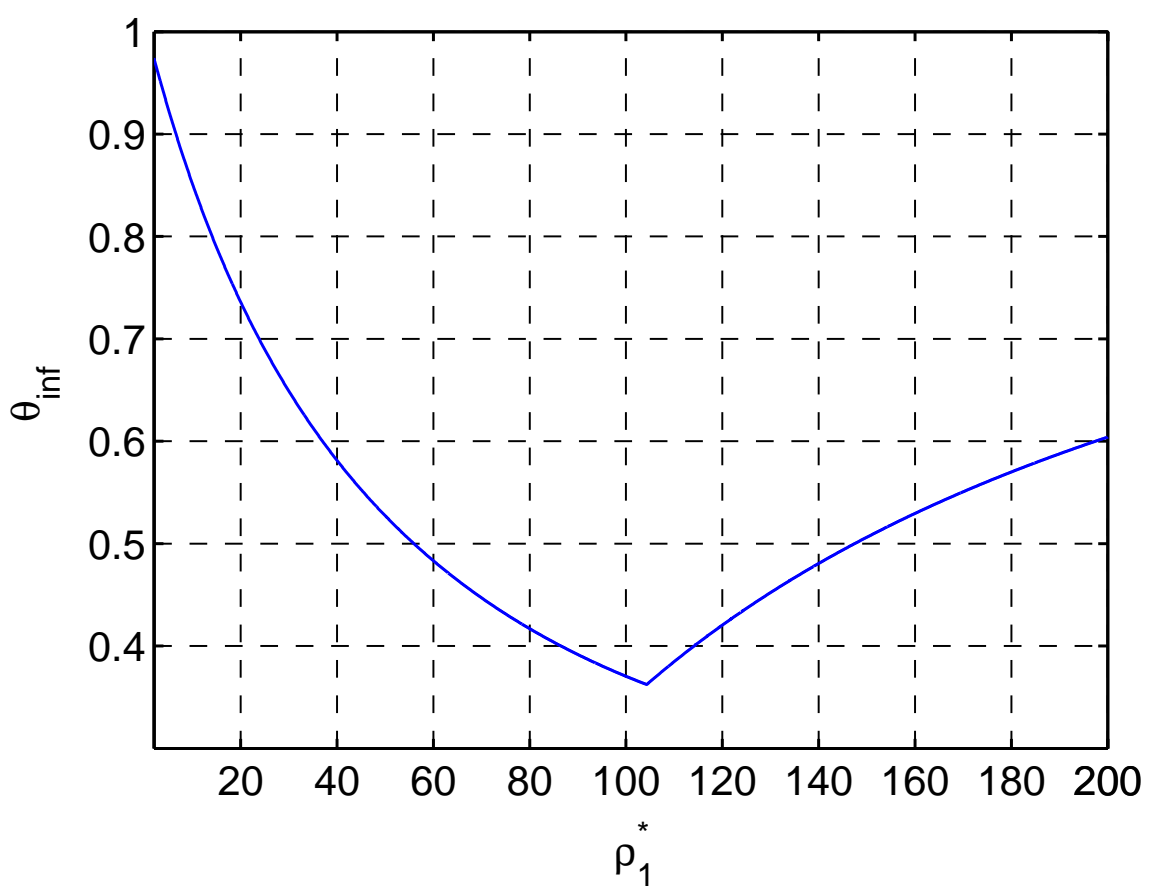

Fig. 2. The relationship curve between the parameter $\rho_{1}^{*}$ and the control rate $\theta_{\text {inf }}$. 


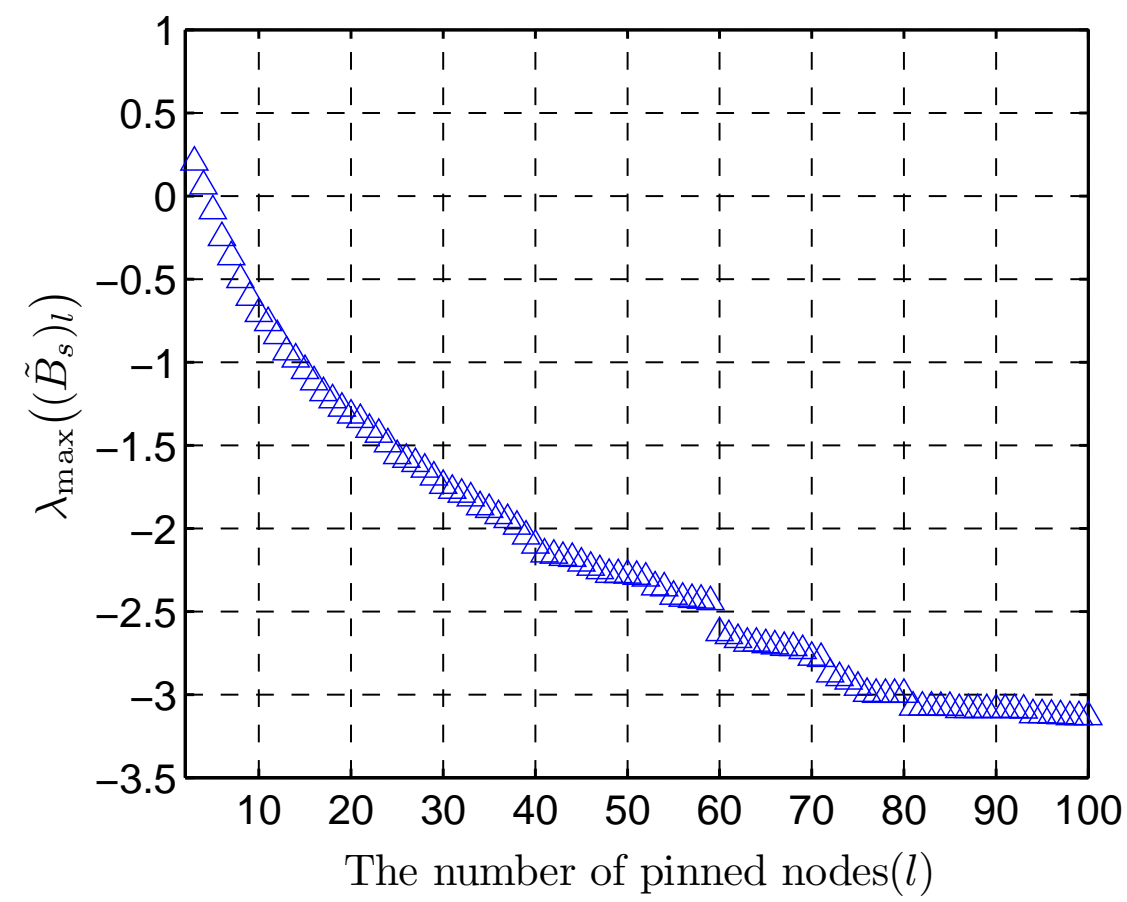

Fig. 3. The number of pinned nodes $(l)$ versus $\lambda_{\max }\left(\left(\tilde{B}_{s}\right)_{l}\right)$.

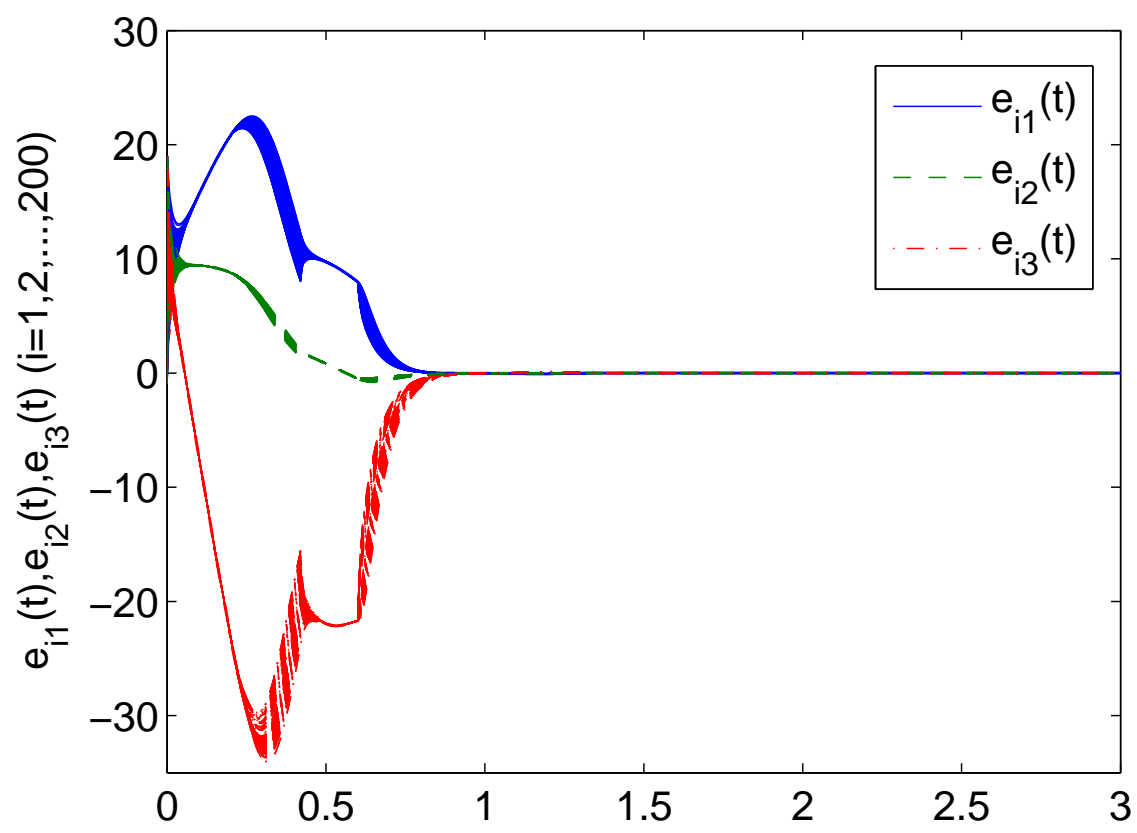

Fig. 4. Time evolutions of synchronous errors $e_{i 1}(t), e_{i 2}(t), e_{i 3}(t), 1 \leq i \leq 200$ between the drive-response networks. 


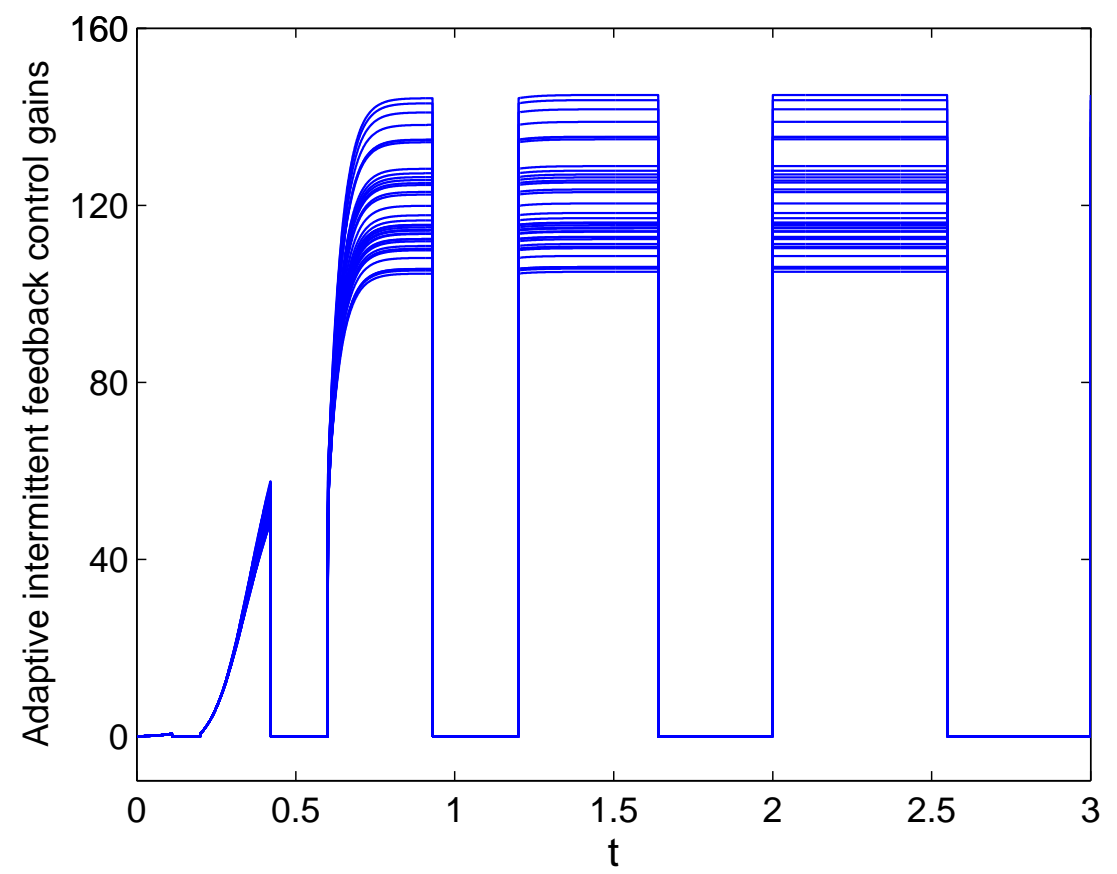

Fig. 5. Time evolutions of adaptive intermittent feedback control gains $d_{i}(t), 1 \leq i \leq 39$. distribution of the Barabási-Albert (BA) network model [49]. The parameters of the BA network model are given by $m_{0}=m=5, N=200$. Let $\kappa_{1}=3$, then one has $K_{1}=11.2083, K_{2}=0.2922$. Since $\varrho_{\min }=1, \varrho_{0}=1.1$ and $\lambda_{\max }\left(\tilde{B}_{s}\right)=0.9116$, we get $\eta_{0}=52.4994$. According to condition (ii') of Corollary 5, the relationship curve between the parameter $\rho_{1}^{*}$ and the control rate $\theta_{\text {inf }}$ is plotted in Fig. 2 . If $\rho_{1}^{*}=50$ is selected as a special case, from conditions ( $\left.\mathrm{i}^{\prime}\right)$ and (ii') of Corollary 5, we can obtain that

$$
\lambda_{\max }\left(\left(\tilde{B}_{s}\right)_{l}\right)<-2.0429 \quad \text { and } \quad 0.5272<\theta_{\text {inf }}<1 .
$$

Using the pinned-node selection scheme for the response network (2) in Remark 4, we rearrange the order of nodes in the response network. Choose $l$ from 1 to 100 and depict the relations between the number of pinned nodes $l$ and $\lambda_{\max }\left(\left(\tilde{B}_{s}\right)_{l}\right)$ in Fig. 3. It can be seen that $\lambda_{\max }\left(\left(\tilde{B}_{s}\right)_{l}\right)$ decreases with the increase of $l$. Especially, when $l=38$ and $l=39$, we have $\lambda_{\max }\left(\left(\tilde{B}_{s}\right)_{38}\right)=-1.9869$ and $\lambda_{\max }\left(\left(\tilde{B}_{s}\right)_{39}\right)=$ -2.0473 . Hence, to realize the outer synchronization, it only needs to choose the 
first $l=39$ rearranged nodes of the response network as pinned nodes.

For numerical simulations, for brevity, we select $\theta_{k} \equiv 0.55, \mathrm{~T}_{k}=t_{k+1}-t_{k}=$ $0.2 k, k \in Z^{+}$and $l=39$, then condition (31) is satisfied. Fig. 5 and Fig. 6 respectively show the evolutions of the synchronous errors and the adaptive intermittent feedback control gains, where the initial conditions are $x_{i}(0)=(-2+0.1 i,-4+$ $0.3 i,-6+0.5 i)^{\top}, y_{i}(0)=(-3+0.2 i,-5+0.4 i,-7+0.6 i)^{\top}$, where $1 \leq i \leq 200$, and $d_{1}(0)=\cdots=d_{39}(0)=0.01, \varepsilon_{1}=\cdots=\varepsilon_{39}=0.001$. It can be seen that the outer synchronization is realized under the aperiodically adaptive intermittent pinning control scheme with $l=39$, and the adaptive intermittent feedback control gains $d_{i}(t), 1 \leq i \leq 39$ intermittently converge to some positive constants. The results of numerical simulations show the correctness of the theoretical analysis.

\section{Conclusion}

In this paper, we have investigated the outer synchronization between two general complex networks with time-varying delayed dynamical nodes via aperiodically adaptive intermittent pinning control. By using the Lyapunov stability theory and rigorous mathematical analysis, some sufficient conditions for outer synchronization are derived through constructing a piecewise auxiliary function. Some simulation results have been provided to show the validity of the theoretical results. Note that, in this paper, only internal delay occurring inside the dynamical node is considered. Since communication delay is ubiquitous in dynamical networks due to the finite speeds of transmission and traffic congestion, our next goal is to study the outer synchronization between two complex dynamical networks with internal delay and communication delay simultaneously via aperiodically adaptive intermittent pinning control. 


\section{Acknowledgements}

The authors sincerely thank the editor and reviewers for their valuable comments and suggestions that helped to improve the content as well as the quality of the manuscript. This work was supported by the National Science Foundation of China (Grant Nos. 11402100 and 11331009), National Science Foundation of China, Tian Yuan Special Foundation (Grant No. 11326193), Natural Science Foundation of Jiangsu Province (Grant No. BK20130535), Young Core Teachers Training Project of Jiangsu University, and the Undergraduate Research Foundation of Jiangsu University (15A004).

\section{References}

[1] S.H. Strogatz, Exploring complex networks, Nature 410 (2001) 268-276.

[2] L.da F. Costa, Jr.O.N. Oliveira, G. Travieso, F.A. Rodrigues, P.R.V. Boas, L. Antiqueira, M.P. Viana, L.E.C. Rocha, Analyzing and modeling real-world phenomena with complex networks: a survey of applications, Adv. Phys. 60 (2011) 329-412.

[3] M.E.J. Newman, The structure and function of complex networks, SIAM Rev. 45 (2003) $167-256$.

[4] X. Wang, G. Chen, Complex networks: small-world, scalefree, and beyond, IEEE Circuits Syst. Mag. 3 (2003) 6-20.

[5] C. W. Wu, Synchronization in Complex Networks of Nonlinear Dynamical Systems, World Scientific, Singapore, 2007.

[6] L.M. Pecora, T.L. Carrol, Master stability function for synchronization coupled systems, Phys. Rev. Lett. 80 (1998) 2109-2112.

[7] X. Wang, G. Chen, Synchronization in small-world dynamical networks, Int. J. Bifurc. Chaos 12 (2002) 187-192. 
[8] J. Lü, G. Chen, A time-varying complex dynamical network model and its controlled synchronization criteria, IEEE Trans. Automat. Control 50 (2005) 841-846.

[9] W. Lu, T. Chen, G. Chen, Synchronization analysis of linearly coupled systems described by differential equations with a coupling delay, Physica D 221 (2006) 118134.

[10] W. Yu, J. Cao, G. Chen, J. Lü, J. Han, W. Wei, Local synchronization of a complex network model, IEEE Trans. Syst., Man, Cybern. Part B: Cybern. 39 (2009) 230-241.

[11] J. Cao, L. Li, Cluster synchronization in an array of hybrid coupled neural networks with delay, Neural Netw. 22 (2009) 335-342.

[12] S. Cai, P. Zhou, Z. Liu, Synchronization analysis of hybrid-coupled delayed dynamical networks with impulsive effects: a unified synchronization criterion, J. Franklin Inst. 352 (2015) 2065-2089.

[13] S. Yamaguchi, H. Isejima, T. Matsuo, R. Okura, K. Yagita, M. Kobayashi, H. Okamura, Synchronization of cellular clocks in the suprachiasmatic nucleus, Science 302 (2003) 1408-1412.

[14] M.I. Rabinovich, P. Varona, A.I. Selverston, H.D.I. Abarbanel, Dynamical principles in neuroscience, Rev. Mod. Phys. 78 (2006) 1213-1265.

[15] C. Li, W. Sun, J. Kurths, Synchronization between two coupled complex networks, Phys. Rev. E 76 (2007) 046204.

[16] J. Wang, J. Zhang, Z. Yuan, A. Chen, T. Zhou, Neurotransmitter-mediated collective rhythms in grouped drosophila circadian clocks, J. Biol. Rhythms 23 (2008) 472-482.

[17] G. Vogel, SARS and bird flu veteran to take WHO helm, Science 314 (2006) 10671067.

[18] H. Tang, L. Chen, J. Lu, C.K. Tse, Adaptive synchronization between two complex networks with nonidentical topological structures, Physica A 387 (2008) 5623-5630. 
[19] S. Zheng, Q. Bi, G. Cai, Adaptive projective synchronization in complex networks with time-varying coupling delay, Phys. Lett. A 373 (2009) 1553-1559.

[20] D. Lin, J. Liu, F. Zhang, Adaptive out synchronization of delay-coupled nonidentical complex networks in the presence of intrinsic time delay and circumstance noise, Nonlinear Dyn. 80 (2015) 117-128.

[21] X. Wu, W. Zheng, J. Zhou, Generalized outer synchronization between complex dynamical networks, Chaos 19 (2009) 013109.

[22] M. Asheghan, J. Míguez, M. T. Hamidi-Beheshti, M. Tavazoei, Robust outer synchronization between two complex networks with fractional order dynamics, Chaos 21 (2011) 033121.

[23] X. Wu, H. Lu, Outer synchronization of uncertain general complex delayed networks with adaptive coupling, Neurocomputing 82 (2012) 157-166.

[24] Y. Sun, D. Zhao, Effects of noise on the outer synchronization of two unidirectionally coupled complex dynamical networks, Chaos 22023131 (2012)

[25] W. Sun, S. Wang, G. Wang, Y. Wu, Lag synchronization via pinning control between two coupled networks, Nonlinear Dyn. 79 (2015) 2659-2666.

[26] Z. Wu, G. Chen, X. Fu, Outer synchronization of drive-response dynamical networks via adaptive impulsive pinning control, J. Frankl. Inst. 352 (2015) 4297-4308.

[27] C. Li, G. Feng, X. Liao, Stabilization of nonlinear systems via periodically intermittent control, IEEE Trans. Circuits Syst. II 54 (2007) 1019-1023.

[28] C. Hu, J. Yu, H. Jiang, Z. Teng, Exponential lag synchronization for neural networks with mixed delays via periodically intermittent control, Chaos 20 (2010) 023108.

[29] C. Hu, J. Yu, H. Jiang, Z. Teng, Exponential synchronization of complex networks with finite distributed delays coupling, IEEE Trans. Neural Netw. 22 (2011) 1999-2010. 
[30] J. Xing, H. Jiang, C. Hu, Exponential synchronization for delayed recurrent neural networks via periodically intermittent control, Neurocomputing 113 (2013) 122-129.

[31] W. Xia, J. Cao, Pinning synchronization of delayed dynamical networks via periodically intermittent control, Chaos 19 (2009) 013120.

[32] S. Cai, J. Hao, Q. He, Z. Liu, Exponential synchronization of complex delayed dynamical networks via pinning periodically intermittent control, Phys. Lett. A 375 (2011) 1965-1971.

[33] S. Cai, P. Zhou, Z. Liu, Pinning synchronization of hybrid-coupled directed delayed dynamical network via intermittent control, Chaos 24 (2014) 033102.

[34] X. Liu, T. Chen, Cluster synchronization in directed networks via intermittent pinning control, IEEE Trans. Neural Netw. 22 (2011) 1009-1020.

[35] S. Cai, P. Zhou, Z. Liu, Intermittent pinning control for cluster synchronization of delayed heterogeneous dynamical networks, Nonlinear Anal. Hybrid Syst. 18 (2015) $134-155$.

[36] X. Liu, P. Li, T. Chen, Cluster synchronization for delayed complex networks via periodically intermittent pinning control, Neurocomputing 162 (2015) 191-200.

[37] C. Hu, H. Jiang, Pinning synchronization for directed networks with node balance via adaptive intermittent control, Nonlinear Dyn. 80 (2015) 295-307.

[38] S. Cai, Q. Jia, Z. Liu, Cluster synchronization for directed heterogeneous dynamical networks via decentralized adaptive intermittent pinning control, Nonlinear Dyn. 82 (2015) 689-702.

[39] J. Mei, M. Jiang, X. Wang, J. Han, an S. Wang, Finite time synchronization of driveresponse systems via periodically intermittent adaptive control, J. Frankl. Inst. 351 (2014) 2691-2710. 
[40] M. Zhao, H. Zhang, Z. Wang, H. Liang, Synchronization between two general complex networks with time-delay by adaptive periodically intermittent pinning control, Neurocomputing 144 (2014) 215-221.

[41] X.-H. Ma, J.-A. Wang, Pinning outer synchronization between two delayed complex networks with nonlinear coupling via adaptive periodically intermittent control, Neurocomputing, 199 (2016) 197-203.

[42] X. Liu, T. Chen, Synchronization of complex networks via aperiodically intermittent pinning control, IEEE Trans. Autom. Control, 60(2015) 3316-3321.

[43] X. Liu, T. Chen, Synchronization of nonlinear coupled networks via aperiodically intermittent pinning control, IEEE Trans. Neural. Netw. Learn. 26 (2015) 113-126.

[44] W. Zhang, C. Li, T. Huang, M. Xiao, Synchronization of neural networks with stochastic perturbation via aperiodically intermittent control, Neural Netw. 71 (2015) 105-111.

[45] X. Liu, T. Chen, Synchronization of linearly coupled networks with delays via aperiodically intermittent pinning control, IEEE Trans. Neural. Netw. Learn. 26 (2015) 2396-2407.

[46] X. Liu, Y. Liu, L. Zhou, Quasi-synchronization of nonlinear coupled chaotic systems via aperiodically intermittent pinning control, Neurocomputing 173 (2016) 759-767.

[47] Q. Song, J. Cao, On pinning synchronization of directed and undirected complex dynamical networks, IEEE Trans. Circuits Syst. -I 57 (2010) 672-680.

[48] J. Zhou, J. Lu, J. Lü, Pinning adaptive synchronization of a general complex dynamical network, Automatica 44 (2008) 996-1003.

[49] A.L. Barabási, R. Albert, Emergence of scaling in random networks, Science 286 (1999) 509-512. 